\title{
Controlling energy transfer in ytterbium complexes: oxygen dependent lanthanide luminescence and singlet oxygen formation
}

\author{
Andrew Watkis, ${ }^{\text {a }}$ Rebekka Hueting, ${ }^{\mathrm{a}}$ Thomas Just Sørensen, ${ }^{\mathrm{b}}$ Manuel Tropiano and Stephen \\ Faulkner ${ }^{\mathrm{a}, *}$
}

\author{
${ }_{5}$ Received (in $\left.X X X, X X X\right)$ Xth $X X X X X X X X X 200 X$, Accepted Xth XXXXXXXXX 200X \\ First published on the web Xth $X X X X X X X X X 200 X$ \\ DOI: $10.1039 / \mathbf{b 0 0 0 0 0 0 x}$
}

\begin{abstract}
Pyrene-appended ytterbium complexes have been prepared using Ugi reactions to vary the chromophore-lanthanide 10 separation. Formation of the ytterbium (III) excited state is sensitised via both the singlet and triplet excited states of the chromophore. Energy transfer from the latter is relatively slow, and gives rise to oxygen-dependent luminescence.
\end{abstract}

Lanthanide luminescence has proved to be an exceptionally 15 useful tool in assay and imaging. ${ }^{1}$ The long-lived luminescence from lanthanide ions can be separated from autofluorescence and scatter using time gating techniques, ${ }^{2}$ and such approaches allow low detection limits and high signal-noise ratios to be achieved.

20 Extensive effort has been devoted to the synthesis of responsive lanthanide complexes, ${ }^{3}$ and a wide range of complexes have been shown to respond to a diverse range of analytes. Since f-f transitions have low molar absorption coefficients, aryl chromophores or transition metal complexes 25 are frequently used to sensitise the formation of an excited state. ${ }^{4}$ While early assays exploited the assembly of ternary complexes to achieve such sensitisation, ${ }^{5}$ more recent work has involved perturbing the fate of the various intermediate excited states. ${ }^{6}$

30 For the vast majority of chromophore appended lanthanide complexes, sensitisation takes place by energy transfer to the lanthanide emissive state from the excited singlet state of the chromophore $\left(\mathrm{S}_{1}\right)$ via the chromophore triplet state $\left(\mathrm{T}_{1}\right){ }^{4}$ It should be noted that energy transfer has been observed from 35 the singlet state, ${ }^{7}$ while a sequential charge transfer mechanism has also been implicated in the sensitised luminescence from ytterbium. All intermediate states in the luminescence pathway can potentially be perturbed by an analyte. Thus collisional quenching of the $S_{1}$ state by halide 40 will reduce the intensity of lanthanide luminescence, ${ }^{8}$ while changes in the inner coordination sphere of the complex will influence the lifetime and intensity of luminescence from the lanthanide excited state. ${ }^{9}$ Where there is a close energy match between the $T_{1}$ state and the lanthanide emissive state that 45 allows thermal repopulation of the triplet, collisional quenching of the $T_{1}$ state by oxygen can give rise to oxygen dependent lanthanide luminescence. ${ }^{10}$ Such an approach has been widely discussed in the development of lanthanide probes for oxygen concentration.

50 An alternative approach to generating oxygen dependent lanthanide luminescence can also be conceived. We recently reported how slow thermal repopulation of a pyrene triplet state from the ${ }^{5} \mathrm{D}_{0}$ state of europium (III), combined with slow (albeit not rate determining) energy transfer from the $T_{1}$ state ${ }_{55}$ to ${ }^{5} \mathrm{D}_{0}$ gave rise to oxygen-dependent lanthanide luminescence as a consequence of collisional quenching occurring before lanthanide luminescence could take place. ${ }^{11}$ This approach can be extended to bimetallic complexes, giving rise to ratiometric responses for oxygen in cases where the rates of energy ${ }_{60}$ transfer to two different lanthanides are different. ${ }^{12}$ At the time, we suggested that such a pathway could give rise to oxygen-responsive luminescence even where thermal repopulation of the triplet was not thermodynamically feasible. In this manuscript we show that this is indeed the ${ }_{65}$ case, and that thermal repopulation is not an essential requirement for oxygen-dependent lanthanide luminescence.

We now report how irreversible energy transfer from a pyrene chromophore to ytterbium can give rise to oxygen dependent luminescence. Furthermore, we have observed the 70 formation of ${ }^{1} \mathrm{O}_{2}$ through its luminescence at $1270 \mathrm{~nm}$. In the systems studied, we observe two pathways for forming the ${ }^{2} \mathrm{~F}_{5 / 2}$ state of $\mathrm{Yb}^{3+}$ that occur simultaneously, with both the singlet state and the triplet state populating the lanthanide emissive state.

The structurally related complexes Yb.1 and Yb.2 were chosen to test the hypothesis of controlling the rate of energy transfer by varying the chromophore-lanthanide separation. Initially, the stable complexes $\mathrm{Yb} .3$ and $\mathrm{Yb} .4$ were prepared using established procedures. ${ }^{7 \mathrm{c}, 13}$ Subsequent coupling using ${ }_{80} \mathrm{Ugi}$ methodologies yielded $\mathrm{Yb.1}$ and $\mathrm{Yb.2}$ as shown in Scheme 1, which were characterised by NMR and MALDI mass spectrometry. This approach offers an effective means by which the metal centre and chromophore can be moved around the scaffold relative to one another.

${ }_{85}$ Upon cursory scrutiny of the structures in Scheme 1, it is clear that the lanthanide ion and the chromophore are separated by spacer chains containing the same numbers of atoms, and that the pyrene chromophores in the two complexes differ slightly in that that in Yb.1 is extended by conjugation. However, the 90 interplay between the functional groups appended from the skeleton will define the preferred orientation of the chromophore relative to the lanthanide ion, and will also influence the chromophore/lanthanide separation. In unrelated systems, we have previously observed how bulky substituents 95 can influence the available conformational space in lanthanide complexes prepared by Ugi reactions: ${ }^{14}$ we reasoned that the same factors would also govern the behaviour of this system. 


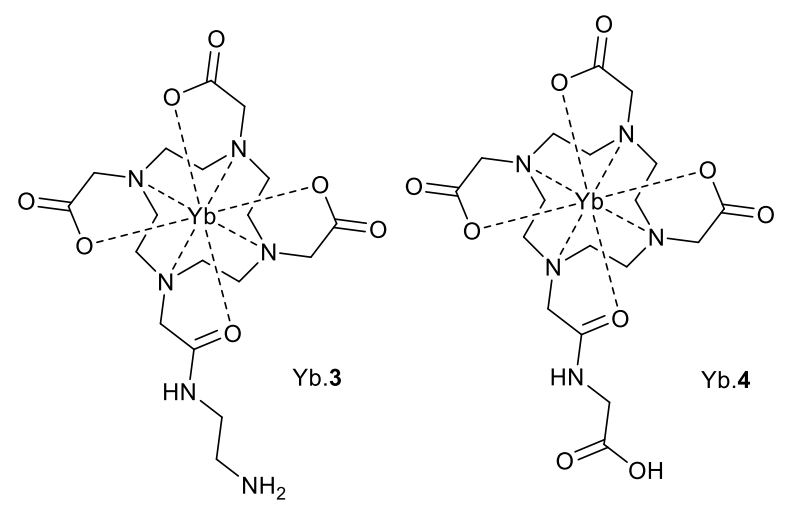<smiles>O=C(O)c1ccc2ccc3cccc4ccc1c2c34</smiles><smiles>O=Cc1ccc2ccc3cccc4ccc1c2c34</smiles>

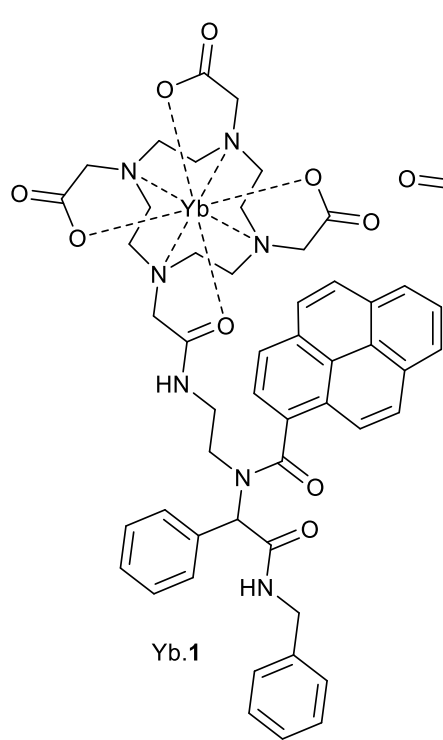

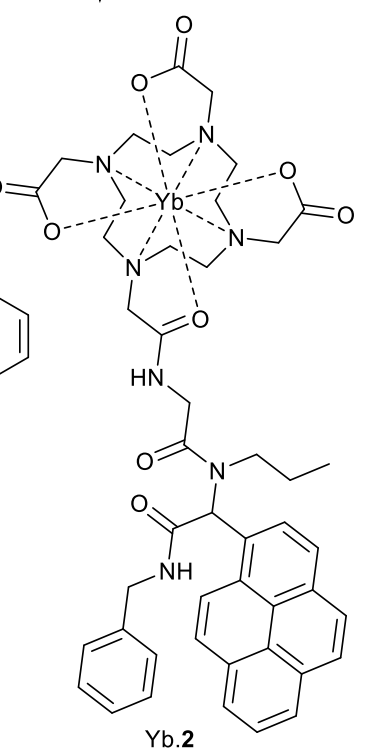

Scheme 1: Preparation of Yb.1 and Yb.2

We resolved to explore this behaviour through luminescence 5 spectroscopy carried out in degassed and aerated solution. Following excitation of the pyrene chromophore at $337 \mathrm{~nm}$, the total emission spectrum of an aerated solution of $\mathrm{Yb} .1$ (Figure 1) revealed the expected peak at $980 \mathrm{~nm}$, corresponding to the ${ }^{2} \mathrm{~F}_{5 / 2}{ }^{2} \mathrm{~F}_{7 / 2}$ transition in $\mathrm{Yb}^{3+}$ and the fine 10 structure associated with crystal field effects, and also showed a second peak at $1270 \mathrm{~nm}$. Upon degassing the sample, the peak at $980 \mathrm{~nm}$ increases in intensity (as a consequence of removal of the oxygen mediated quenching pathway for the donor triplet) while that at $1270 \mathrm{~nm}$ disappears entirely. The

$151270 \mathrm{~nm}$ peak corresponds to the wavelength associated with luminescence by singlet oxygen, ${ }^{10 \mathrm{c}}$ and these results clearly imply formation of ${ }^{1} \mathrm{O}_{2}$ by collisional interactions with the excited triplet state of the complex.

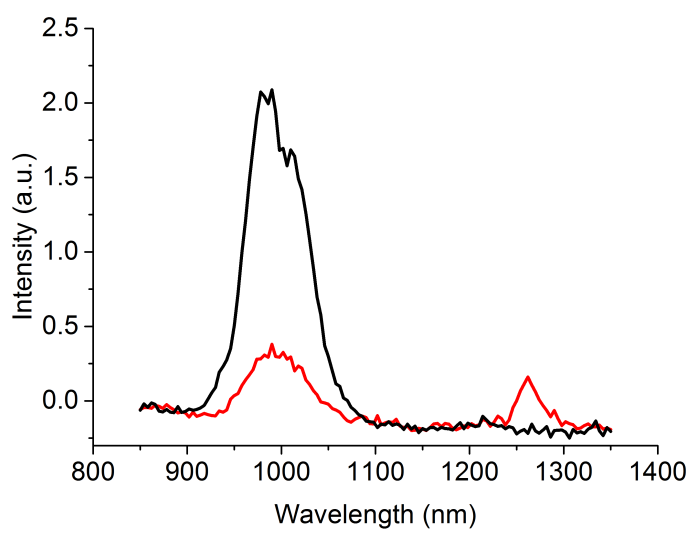

20 Figure 1: near IR emission spectra obtained following excitation at 337 $\mathrm{nm}$ for a methanolic solution of Yb.1 under aerated (red line) and degassed (black line) conditions and showing emission from $\mathrm{Yb}^{2} \mathrm{~F}_{5 / 2}{ }^{2} \mathrm{~F}_{7 / 2}$ $(980 \mathrm{~nm})$ and ${ }^{1} \mathrm{O}_{2}-{ }^{3} \mathrm{O}_{2}(1270 \mathrm{~nm})$.

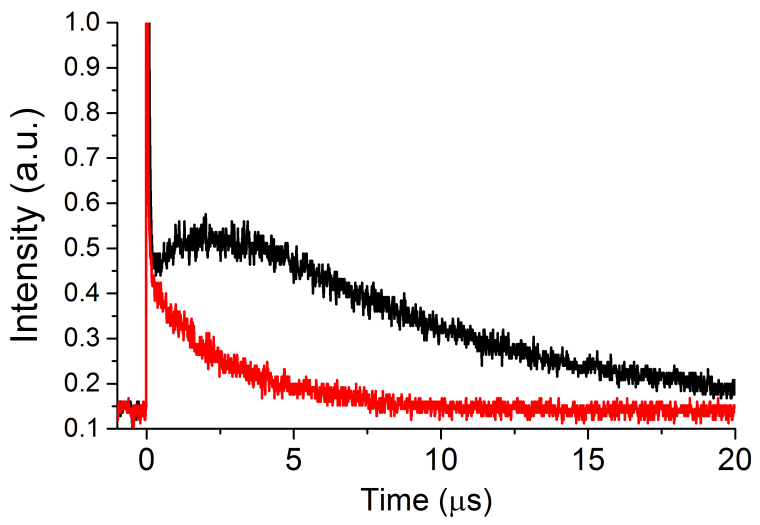

Figure 2: temporal profiles of the emission intensity at $980 \mathrm{~nm}$ for 25 aerated (red line) and degassed (black line) methanolic solutions of Yb.1

Time-resolved measurements shed further light on the nature of energy transfer and ${ }^{1} \mathrm{O}_{2}$ formation. There are clear and dramatic differences between the temporal profiles of the 30 emission from $\mathrm{Yb} . \mathbf{1}$ in degassed and aerated methanolic solution (Fig. 2). In the aerated system, the risetime of the luminescence from ytterbium is essentially instantaneous with a long lived decay component $(\tau=2.8 \mu \mathrm{s})$, while that in degassed solution displays two clear components - in which 35 one clearly involves a relatively slow risetime, superimposed upon the aerated response.

The luminescence lifetime of the $1270 \mathrm{~nm}$ band correspnding to ${ }^{1} \mathrm{O}_{2}$ emission $(10 \mu \mathrm{s})$ corresponds very closely with the published value for singlet oxygen in methanol. ${ }^{15}$ In degassed 40 solution, energy transfer from $\mathrm{T}_{1}$ to the ${ }^{2} \mathrm{~F}_{5 / 2}$ excited state of ytterbium is rate determining (Scheme 2). In these circumstances ${ }^{7 \mathrm{a}}$ the decay component $(\tau=7.4 \mu \mathrm{s})$ corresponds to the rate of energy transfer, while the rise-time $(\tau=2.1 \mu \mathrm{s})$ corresponds to the lanthanide centred emission. 


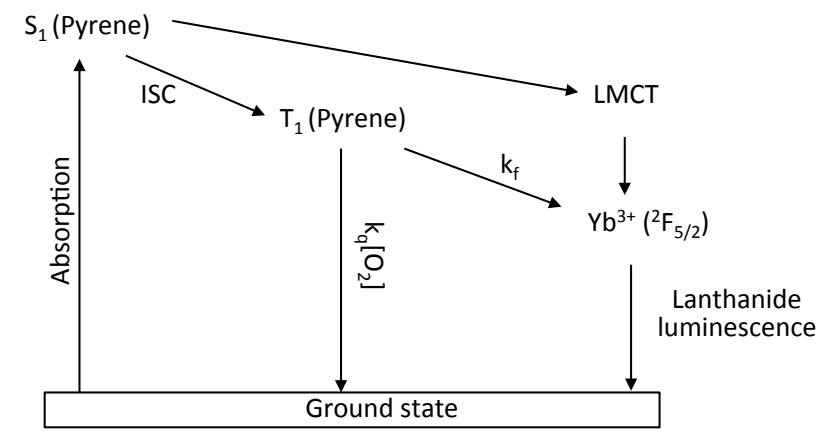

Scheme 2: Key energy transfer pathways in an ytterbium complex bearing a pyrenyl antenna group.

It is clear that two pathways contribute to the formation of 5 the lanthanide excited state (Scheme 2): the $S_{1}$ and $T_{1}$ states of the pyrene chromophore are both involved in energy transfer, albeit by different routes. In the case of the $\mathrm{S}_{1}$ state, the most likely pathway is a sequential electron transfer mechanism going via an LMCT state, which would be expected to give ${ }_{10}$ rise to rapid energy transfer. ${ }^{7 a}$ By contrast, the rate of triplet mediated energy transfer will be slow as a consequence of the poor spectral overlap between $\mathrm{T}_{1}$ and ${ }^{2} \mathrm{~F}_{5 / 2}$. Collisional quenching by oxygen can thus quench the intermediate triplet state before energy transfer to the lanthanide centre, lowering 15 the luminescence quantum yield.

We have previously observed such phenomena in systems in which there is a close energy match between the donor state and the accceptor state, combined with a large physical separation between the chromophore and the lanthanide ion. ${ }^{11}$

20 In this case the energy gap between the $T_{1}$ state $\left(E_{T}=17,300\right.$ $\left.\mathrm{cm}^{-1}\right)$ and the ${ }^{2} \mathrm{~F}_{5 / 2}$ state $\left(\mathrm{E}_{2 \mathrm{~F} 5 / 2}=10,300 \mathrm{~cm}^{-1}\right)$ is too great to permit thermal repopulation of the triplet. It is therefore clear that a close match between the triplet energy and the lanthanide emissive state is not a pre-requisite for achieving 25 oxygen-dependent lanthanide emission, provided slow energy transfer can be engineered into the system.

In the case of $\mathrm{Yb.2}$, the observed spectra were very different. In degassed solution, luminescence from the ytterbium centre was almost too weak to detect using steady 30 state luminescence- indeed, the ${ }^{2} \mathrm{~F}_{5 / 2}-{ }^{2} \mathrm{~F}_{7 / 2}$ transition could only be resolved by applying time-gating methods to separate the ytterbium signal from scattered light (Figure S7). However, in aerated media, strong phosphorescence from singlet oxygen was oberved at $1270 \mathrm{~nm}$, indicating that the triplet state of the 35 chromophore is still quenched effectively by oxygen. It is clear from these observations that the difference in structure between $\mathrm{Yb} .1$ and $\mathrm{Yb} .2$ is responsible for the difference in observed behaviour and that differences in the effectiveness of energy transfer are underpinned by differences in the relative 40 orientation of donor and acceptor groups. Once again, it is clear that ligand structure is key to controlling the photophysical properties.

On the basis that the observation of emission from ${ }^{1} \mathrm{O}_{2}$ in aerated systems could potentially offer a second signal that 45 would allow quantification of oxygen levels, we explored the variation in relative intensity of the lanthanide and oxygen centred emission bands with oxygen concentration for solutions containing Yb.1. Figure 3 shows that there is a clear variation in the ratio of the two intensities with oxygen, ${ }_{50}$ confirming the hypothesis of competitve quenching of the intermediate triplet state and also providing the potential for quantifying oxygen concentration.

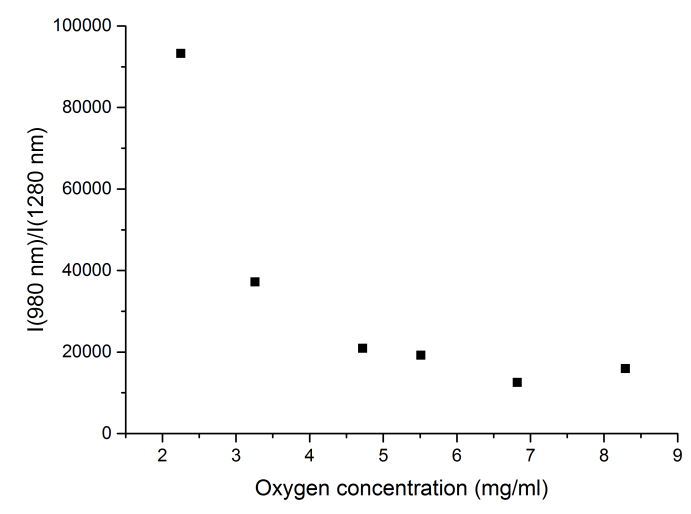

Figure 3: Oxygen dependence of the ratio of the intensity of the ytterbium ${ }^{2} \mathrm{~F}_{5 / 2}-{ }^{2} \mathrm{~F}_{7 / 2}$ transition $\left(980 \mathrm{~nm}\right.$ ) relative to that of the ${ }^{1} \mathrm{O}_{2}-{ }^{3} \mathrm{O}_{2}$ emission intensity $(1270 \mathrm{~nm})$.

It is clear from these results that ${ }^{1} \mathrm{O}_{2}$ emission and NIR ${ }_{60}$ lanthanide emission can be combined to provide a ratiometric measure of oxygen concentration. This approach has considerable merit, offering the possibility of combining straightforward synthesis with effective measurement and allowing steady state measurements to be used to quantify 65 oxygen concentrations directly. There is clearly scope to extend these results to other sensitising chromophores, and indeed longer wavelength excitation is highly desirable. We also add a general caveat that distance dependence and rates need to be considered for all the steps in the energy transfer 70 cascade for a lanthanide complex; it clearly isn't enough to assume that the only thing to worry about is the emissive lifetime.

\section{Notes and references}

The authors thank the EPSRC (EP/I021949/2), the Carlsberg 75 and Villum Foundations, and the Universities of Oxford and Copenhagen, together with Keble College and Christ Church for support.

${ }^{a}$ University of Oxford, Chemistry Research Laboratory, 12 Mansfield 80 Road, Oxford OX1 3TA UK Stephen.Faulkner@chem.ox.ac.uk

${ }^{b}$ University of Copenhagen, Chemistry Research Laboratory, 12 Mansfield Road, Oxford OXI 3TA UK

$\dagger$ Electronic Supplementary Information (ESI) available: [details of any 85 supplementary information available should be included here]. See DOI: $10.1039 / \mathrm{b} 000000 \mathrm{x} /$

1 S. V. Eliseeva and J.-C. G. Bünzli, Chem. Soc. Rev., 2010, 39, 189; I. Hemmilä and V. Laitala, J. Fluoresc., 2005, 15, 529.

902 A. Beeby, S. W. Botchway, I. M. Clarkson, S. Faulkner, A. W. Parker, D. Parker and J. A. G. Williams, J. Photochem. Photobiol. $B$, 2000, 57, 83; L. Charbonnière, R. Ziessel, M. Guardigli, A. Roda, N. Sabbatini and M. Cesario, J. Am. Chem. Soc., 2001, 123, 
2436; Z. Liao, M. Tropiano, S. Faulkner, T.Vosch, T. Just Sørensen, RSC Advances, 2015, 5, 70282

3 D. Parker, Chem. Soc. Rev., 2004, 33, 156-165

4 For a review see, S. Faulkner, L. S. Natrajan, W. S. Perry and D. Sykes, Dalton Trans., 2009, 3890

5 I. A. Hemmilä, Applications of fluorescence in immunoassays, Wiley, 1991

6 C.P. Montgomery, B.S. Murray, E.J. New, R. Pal, D. Parker, Acc Chem Res 2009, 42, 925

107 a) A. Beeby, S. Faulkner and J. A. G. Williams, J. Chem. Soc., Dalton Trans., 2002, 1918; b) T. Lazarides, M.A.H. Alamiry, H Adams, S.J.A. Pope, S. Faulkner, J.A. Weinstein \& M.D. Ward, Dalton Trans., 2007, 1484-1491; c) S.J.A. Pope, W.S. Perry, C. Allain, A.M. Kenwright and S. Faulkner, Dalton Trans. 2010, 2010, 39, 10974

8 S.J. Butler, D. Parker Chem Soc Rev, 2013, 42, 1652

9 R.Pal, D.Parker, L.C. Costello, Org. Biomol. Chem., 2009, 7, 1525

10 a) A. Beeby, D. Parker and J.A.G. Williams, J. Chem. Soc., Perkin Trans. 2, 1996, 1565-1579 b) G. L. Law, R. Pal, L. O. Palsson, D. Parker and K. L. Wong, Chem. Commun., 2009, 7321; c) A. Beeby, S. Faulkner, D. Parker \& J.A.G. Williams, J. Chem. Soc. Perkin Trans. 2, 2001, 1268; d) X. Wang \& O.S. Wolfbeis Chem. Soc. Rev., 2014,43, 3666

11 R. Hueting, M. Tropiano, S. Faulkner RSC Advances, 2014, 4, 44162

2512 T.J. Sørensen, A.M. Kenwright and S. Faulkner, Chem. Sci. 2015, 6, 2054

13 T. J. Sørensen, M. Tropiano, O. A. Blackburn, J. A. Tilney, A. M. Kenwright and S. Faulkner, Chem. Commun., 2013, 49, 783

14 M. Tropiano, O. A. Blackburn, J. A. Tilney, L. R. Hill, M. P. Placidi, R. J. Aarons, D. Sykes, M. W. Jones, A. M. Kenwright, J. S. Snaith, T. J. Sørensen and S. Faulkner, Chem.-Eur. J., 2013, 19, 16566

15 P.R. Ogilby, Chem. Soc.Rev., 2010, 39, 3181 